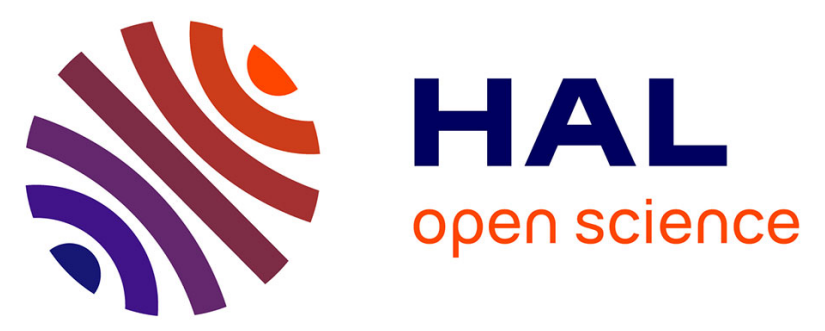

\title{
Characterization of micro/nano-rheology properties of soft and biological matter combined with a virtual reality haptic exploration
}

Claudie Petit, Marwene Kechiche, Ioan-Alexandru Ivan, Rosario Toscano, Valentine Bolcato, Emmanuelle Planus, Florence Marchi

\section{To cite this version:}

Claudie Petit, Marwene Kechiche, Ioan-Alexandru Ivan, Rosario Toscano, Valentine Bolcato, et al.. Characterization of micro/nano-rheology properties of soft and biological matter combined with a virtual reality haptic exploration. 2019 International Conference on Manipulation, Automation and Robotics at Small Scales (MARSS), Jul 2019, Helsinki, Finland. 10.1109/MARSS.2019.8860978 . hal-02141911

\section{HAL Id: hal-02141911 \\ https://hal.science/hal-02141911}

Submitted on 28 May 2019

HAL is a multi-disciplinary open access archive for the deposit and dissemination of scientific research documents, whether they are published or not. The documents may come from teaching and research institutions in France or abroad, or from public or private research centers.
L'archive ouverte pluridisciplinaire HAL, est destinée au dépôt et à la diffusion de documents scientifiques de niveau recherche, publiés ou non, émanant des établissements d'enseignement et de recherche français ou étrangers, des laboratoires publics ou privés. 


\title{
Characterization of micro/nano-rheology properties of soft and biological matter combined with a virtual reality haptic exploration
}

\author{
Claudie Petit ${ }^{1}$, Marwene Kechiche ${ }^{2}$, Ioan-Alexandru Ivan ${ }^{2}$, Rosario Toscano ${ }^{2}$, \\ Valentine Bolcato ${ }^{3}$, Emmanuelle Planus ${ }^{4}$, and Florence Marchi ${ }^{5}$
}

\begin{abstract}
Science education is often limited by the complexity of making acute knowledge accessible and easily remembered by a larger public. We proposed another way to introduce and teach complex scientific subjects like cell biology and polymer micro-rheology using haptic display and virtual reality. We combined the advantages of the Atomic Force Microscopy (AFM) and Fluorescence Microscopy (FM) in order to get complementary real experimental data on an isolated animal adhering on a protein micro-pattern. We obtained high resolution mapping images of the cell morphology, architecture, and local mechanical properties. Then this set of data was implemented in a free simulation engine connected to a low-cost haptic device to create a virtual and interactive cell or polymer environment in order to provide a novel sensory approach of the cell biology and/or micro(bio)mechanics.
\end{abstract}

\section{INTRODUCTION}

In the past twenty years, several studies in cellular biology have reveals the role of the mechanical environment in cell development and rheological properties [1]. Three key examples illustrate this aspect : (1) The value of the stiffness of the adhesive substrate impacts the stem cell differentiation [2]; (2) The cells generate forces to shape the tissues during morphogenesis. The cell-cell interaction involves mechanically regulated clusters allowing the tensile forces to be coupled and transmitted during gastrulation and tissue polarization [3] ; (3) Atomic Force Microscope (AFM) nanoindentation on living healthy and tumorigenic cells revealed the effect of their different cytoskeletal conformations on cell stiffness [4]. This leads to a possible additional way to distinguish between cancer and healthy tissues. Based on these major discoveries highlighting that genetics cannot explain the whole development of cell, a new research field based on the concept of mechano-chemical transduction is growing to tackle these cell-environment interactions and their role in biological cells and tissues development [5]. In this context, it appears crucial to developed new concepts

1. Petit was with CIME Nanotech and is now with EMSE, Univ Lyon, Univ Jean Monnet, INSERM, U 1059 SAINBIOSE, Saint-Etienne, France claudie.petitdemse. fr

${ }^{2}$ M. Kechiche, I.A. Ivan and R. Toscano are with Ecole Nationale d' Ingénieurs de Saint-Etienne, ENISE, Univ Lyon, LTDS, CNRS UMR 5513 France ivandenise.fr

$3 \mathrm{~V}$. Bolcato is with CIME Nanotech, Grenoble INP, University of Grenoble Alpes, France valentine.bolcato@grenoble-inp. fr

${ }^{4} \mathrm{E}$. Planus is with UFR de Chimie et de Biologie, Univ Grenoble Alpes, UGA, Institute for Advanced Bioscience, CNRS UMR 5309, La Tronche France, France emmanuelle.Planuscuniv-grenoble-alpes.fr

* 5 F. Marchi is with UFR PhITEM, Univ Grenoble Alpes, UGA, Institut Néel, CNRS/UGA UPR 2940, Grenoble France, France florence.marchi@neel. chrs.fr of teaching and training in order to illustrate the impact of the environment on rheological properties and cell morphology at micro and nanoscales. This new concept should be adaptable to the scientific and technical background and level of various students and pupils as well as the time dedicated to the practical and/or discovering activities. The main goals of these educational activities are threefold : (1) To perform the culture of mouse osteoblast cells and to seed them on a CYTOO ${ }^{T M}$ chip where an adhesive fibronectin (FN) micropattern array is printed on a glass substrate surrounded by a cytophobic layer; In this condition, the cells face a spatial environmental stress where they have to modulate their internal mechanical response in order to fit their morphology; (2) To introduce how to use the main and complementary characterization techniques involved in this kind of micro/nano-rheology studies such as AFM and fluorescence microscopy (FM) and how to combine them to enhance the information display; (3) To provide an intuitive mainstream access to the biomechanics of a cell. To reach this last goal, we have developed a virtual and interactive nano scene based on AFM experimental data recorded with one of the most popular AFM mode in micro/nano-rheology field called «Peakforce»(PF). This nanoscene displays visual and haptics renderings leading to an interactive offline exploration of the stiffness variation along a spread mouse osteoblast. Indeed, previous studies have successfully demonstrated that virtual reality combining visual rendering and haptic feedback is very powerful and well adapted to provide a quick and deep understanding of phenomena that remain out of human perception, as molecular bonding [6] ; [7], surgery [8], cell biology [9], [10] or micro/nano-scale forces taking place between a AFM tip and a sample [11]. The AFM probe is able to measure local force interactions with a sub-nanoNewton resolution at nanoscale using the so-called force spectroscopy mode [12]. When this force measurement is performed with a scanning movement and an automatic feedback loop, the AFM probe acts as a nanorobot and leads at the same time to 3D maps of morphology and force maps; this topography and force combination was firstly called «Force-volume » $(\mathrm{FV})$ mode. In the past this mode was in some cases performed on single fixed or living cell to map its response to a mechanical stress [13], [14]. However this process was time-consuming (a whole combined maps with a high resolution require several hours), it was not a routine mode. Recently a much faster version of this mode called «Peakforce » $(\mathrm{PF})$ was implemented by Bruker company in their last version of AFM instruments 
[15], stimulating new trends in cell biology [16]; [17]. Today Peakforce mode becomes the standard AFM mode in this research field. The development, the first of our knowledge, where a virtual nanoscene based experimental morphology and force maps recorded on a single cell in PF mode is coupled with a Haptic device, is described in the last part of this paper (section IV). This virtual and interactive station offers the opportunity to explore the morphology and local stiffness variation of the main cellular components on a fixed through our human sensory capabilities, as if we were able to see them through our eyes and to touch them with a nanofinger to feel their elasticity. The section II is dedicated to cell sample preparation and to the spatial identification of its main components by combining fluorescent and AFM microscopies. The section III covers the morphological and mechanical characterizations of soft samples by AFM Peakforce mode.

\section{Cell SAmple Preparation and Identification}

A mouse osteoblast cell line developed internally at the Institute for Advanced Biosciences (IAB) from Grenoble was used for this study. Cells were cultured in plastic dish formerly according to a standard protocol using complete medium containing Dulbecco's modified Eagle's medium (DMEM) with high glucose $(4.5 \mathrm{~g} / \mathrm{L}), 10 \%$ Fetal Bovine Serum (FBS) and Penicillin-Streptomycin antibiotic mix (PS). Cells were seeded at the density of $6.10^{4}$ cells per CYTOO $^{T M}$ Starter chips according to the manufacturer's instruction. After 2 hours incubation, cells were well spread and cell samples components were fluorescently stained for FM characterization (Fig. 1). The standard protocol for fluorescent staining consists in three steps : paraformaldehyde (4\%) fixation, plasma membrane mild permeabilization with Triton X100, and staining with regular immunofluorescence $(\mathrm{ABs})$ procedure.

Once stained, the cell samples remained hydrated in Phosphate Buffered Saline (PBS) supplemented with PS for further characterization in FM and AFM. For FM we used a Carl Zeiss LSM 800 microscope for the identification of the different cell components constituting the cell architecture. Since the cell architecture may present various stiffness values on its whole surface during indentation, it is relevant to locate and identify the main cell components, namely : the cytoskeleton (CSK), made of filamentous act in (F-Actin) (Fig. 1C), the Focal Adhesions (FA) (Fig. 1D) linking the cell's CSK to the underlying extracellular matrix (ECM), and the nucleus (Fig. 1E), the largest organelle which contains the genetic information in the DNA. The advantage of the CYTOO chips is to provide a fluorescent grid with several markers which are coded (Fig. 1A), so that the same single cell can be spotted for successive FM and AFM characterizations in PBS [14]. This combination of characterization techniques is fundamental for a better understanding of the organization and localization of the main cell components down to the nanoscale.

\section{MORPHOLOGICAL AND MECHANICAL CHARACTERIZATION BY AFM}

\section{A. Atomic Force Microscopy (AFM)}

he AFM technique is a morphology and nanomechanical sensing method which was rewarded with a Nobel Prize in 1986 [18]; [19]. The technique consists of a topographic
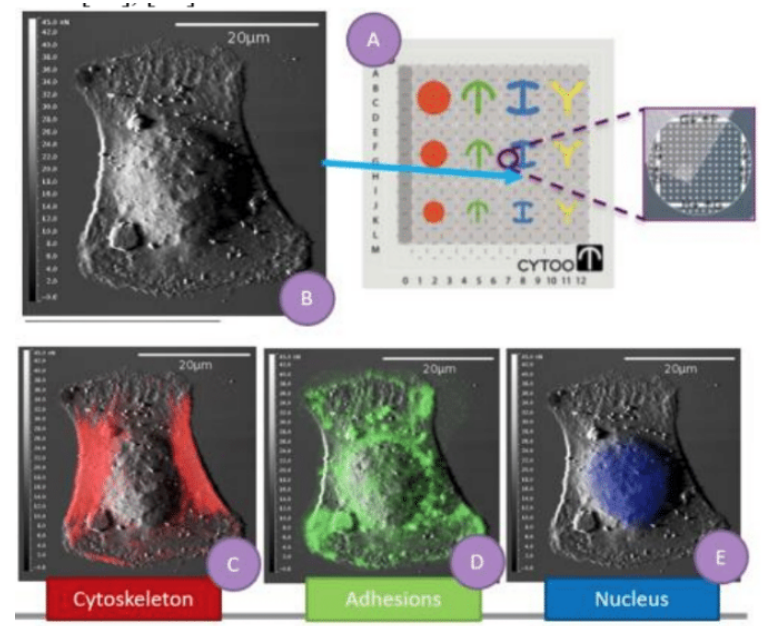

Fig. 1. (A) The CYTOO chip consists of a glass coverslide with imprinting FN micropatterns where the cells adhere individually. The micropatterns are disposed in an array of squares, each square is labelled by a couple of letters and numbers to facilitate their localization (insert in (A)). (B) A osteoblast cell fixed on an $I$ micropattern characterized by AFM (the gray profile) in liquid. Then the same cell is chosen and characterized in liquid by FM to identify and to localize in the volume the different cell components, namely : The F-actin cytoskeleton (C), the focal adhesions (D) and the nucleus (E).

analysis over a small specified area by raster-scanning the surface with a very sharp tip at the end of a flexible cantilever (Fig. 2). This method is suitable for any type of material, because it relies only on the interatomic interaction, long-range forces (electromagnetic, electrostatic) and shortrange ones (chemical, capillarity, Van der Walls) [19]. The combination of all these effects leads to a distance-dependent potential between the atoms of the AFM tip and those of the sample surface. Hence, this interaction induces a deflection on the cantilever during the scan (Fig. 2E). The mechanical behavior of the cantilever is associated to its spring constant $k(\mathrm{~N} / \mathrm{m})$, which can be well estimated thanks to the wellestablished Brownian motion method [20]. In practice, the value of the spring constant should be carefully selected according to the intensity of tip-sample force needed to scan the sample without damage (i.e. low $k$ for soft and fragile sample as biological cell). The AFM is a powerful tool to map the topography (Fig. 2F) but also to various mechanical, electrical and even magnetic quantities [19] at nanoscale. Several different AFM working modes provide a topographic characterization, including the so-called contact and tapping modes [21]. The contact mode maintains a constant interaction by keeping the cantilever deflection equal to the setpoint (i.e., the reference deflection set in the scan parameters). On the contrary, the tapping mode results in an intermittent interaction between the tip and sample; 
that is the reason why it is applied preferably for sensitive samples like biological ones. Nevertheless, none of them offered a direct and rapid access to the mechanical properties of the sample such as the surface stiffness.

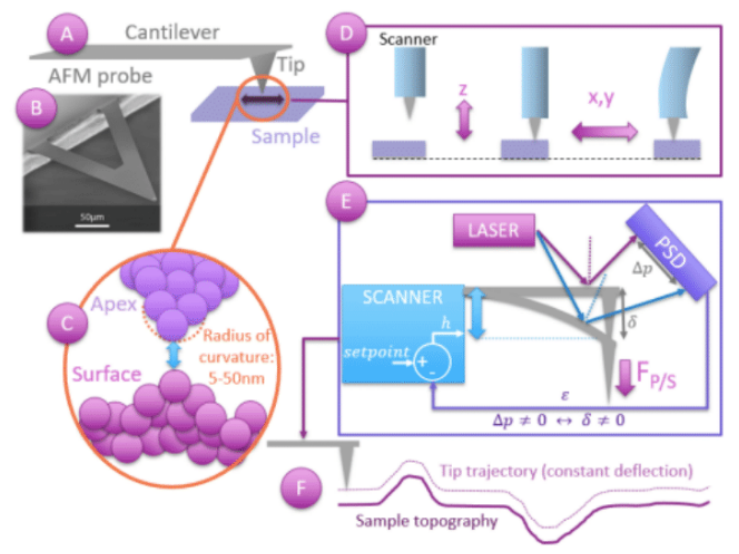

Fig. 2. The AFM probe consists in a sharp silicon tip at the end of a flexible microcantilever (A) and (B). The tip apex is approximated like a nanosphere leading to a nanometric lateral resolution $(\mathrm{C})$, the scan of the sample surface is done using a piezoelectric actuator (D). The interatomic interactions between the apex of the tip and the sample surface induce the deflection of the cantilever $\delta(\mathrm{C})$ and $(\mathrm{E})$. The variation of the deflection during the scan is tracked by an optical detection system where a reflected laser beam over the cantilever top surface that reaches a photosensitive device (PSD) (E). In contact mode, the deflection $\delta$ is regulated according to the chosen setpoint $\Delta p$ in the initial scan parameters $(\mathrm{E})$, resulting to a constant distance between the apex of the tip and the sample surface revealing its topography $(\mathrm{F})$

\section{B. Nanoindentation by AFM}

The AFM probe can act as a nanoindentor to sense the sample local mechanical properties [22]. In the static mode, the tip successively approaches the surface and retracts over a single point (Fig. 3A), while the photosensitive device computes the cantilever deflection $\delta(\mathrm{V})$ according to the scanner vertical displacement $Z s(\mathrm{~nm})$. The result is an approachretract force curve (Fig. 3B). A prior calibration defines the detection sensitivity $s(\mathrm{~nm} / \mathrm{V})$ of the photodetector. As a result, the curve can be plotted as force measurement $F(\mathrm{nN})$ versus the indentation depth $h$ (equations in Fig. 3C and Fig. 3D) [23]. This force curve (Fig. 3B) displays various mechanical measurements such as adhesion force $F_{a d}$ and maximal indentation depth $h_{\text {ind }}$. Then, from the slope of the curve $F$ versus $h$ (Fig. 3B) the contact stiffness $k_{c}$ can be deduced and the local Young Modulus $E$ can be estimated by applying a proper mechanical model of contact [24]. The Force Volume (FV) mode repeats the same operation (Fig. 3) on successive points over a specified area of interest and yields all the associated force curves. Moreover, the intermittent contact avoids high frictional forces of the standard contact mode and so sample damaging. Although this mode was widely used at the beginning of the investigation of the cell mechanics, its quasi-static functioning based on slow triangular signals (less than $10 \mathrm{~Hz}$ ) is time consuming, taking several hours for a full scan or is limited to poor resolutions such as $(32 * 32)$ or $(64 * 64)$ pixels [25]. In this context, the PeakForce mode with its signal acquisition efficiency has been gaining a particular interest in the applications combining AFM and biology, such as topography and rheology of living single-cells.

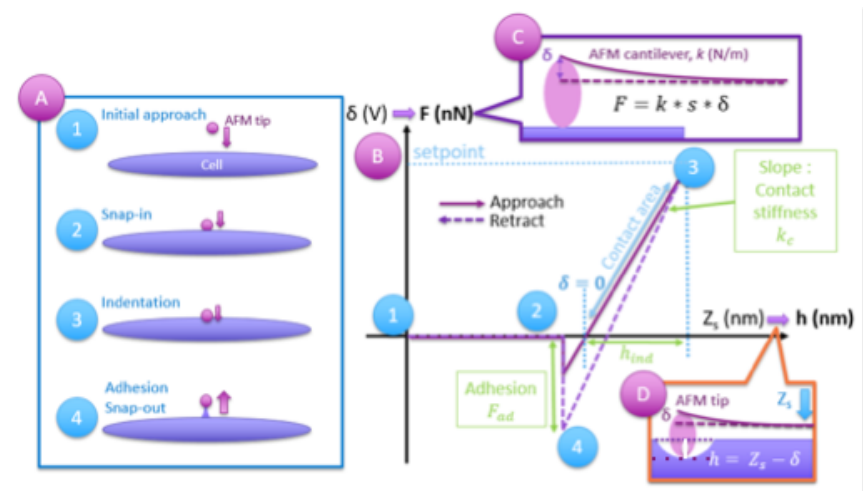

Fig. 3. AFM nanoindentation of a single cell in the static mode over a fixed point. (A) The approach-retract cycle; (B) The associated force curve plotting initially cantilever deflection $\delta$ in function of scanner extension $Z_{s}$ and the the force $F$ according to the tip-sample separation $h$ where $h_{i n d}$ is the maximum indentation depth, which is similar to a classical indentation curve; (C) The force $F$ conversion formula; (D) The indentation depth $h=Z_{s}-\delta$

\section{PeackForce mode}

The PeakForce (PF) mode derives from the FV mode, it records successive force curves thanks to a dynamic sinusoidal signal applied on the piezoelectric actuator to drive quickly the cantilever up and down to the sample (Fig. 4). The cantilever is driven at frequency $f_{P F}$ that can be tuned between 0.25 up to $2 \mathrm{kHz} ; f_{P F}$ should be significantly lower than its resonance frequency $f_{0}$ (Fig. 4B) to drag the cantilever in a steady position. To fit this condition as well as a low cantilever spring constant (to avoid damage of cell sample), we selected a Scanasystliquid AFM probe with measured parameters in air and at room temperature of respectively $84.4 \mathrm{kHz}$ and $0.65 \mathrm{~N} / \mathrm{m}$ for $f_{0}$ and $k$. The acquisition time in PF mode is about few minutes for high resolution $(128 * 128$ or $256 * 256$ pixels) and combined 3D topography and force maps. Moreover, this non-resonant mode implies that the tip follows the movement of the scanner avoiding the dynamics of aresonating system (Fig. 4B). The PF mode therefore combines the advantage of contact mode, namely direct force control, and tapping mode, i.e. intermittent contact avoiding damages due to permanent friction. For direct force control, the PF mode relies on the adjustment of the setpoint that corresponds to the maximal force applied on the sample while acquiring the spectroscopy curves (Fig. 4D). Hence, the height of the scanner is automatically adapted according to the local sample structure in order to keep the setpoint over the entire area of interest. In this way, the PF mode provides both topography and mechanical mapping. The setpoint parameter has to be carefully chosen as the best compromise between good-matching force acquisition and force-time curves (Fig. 3B and Fig. 4D) and without damaging the cell surface due to too high interaction forces. In practice, to map the cell in 


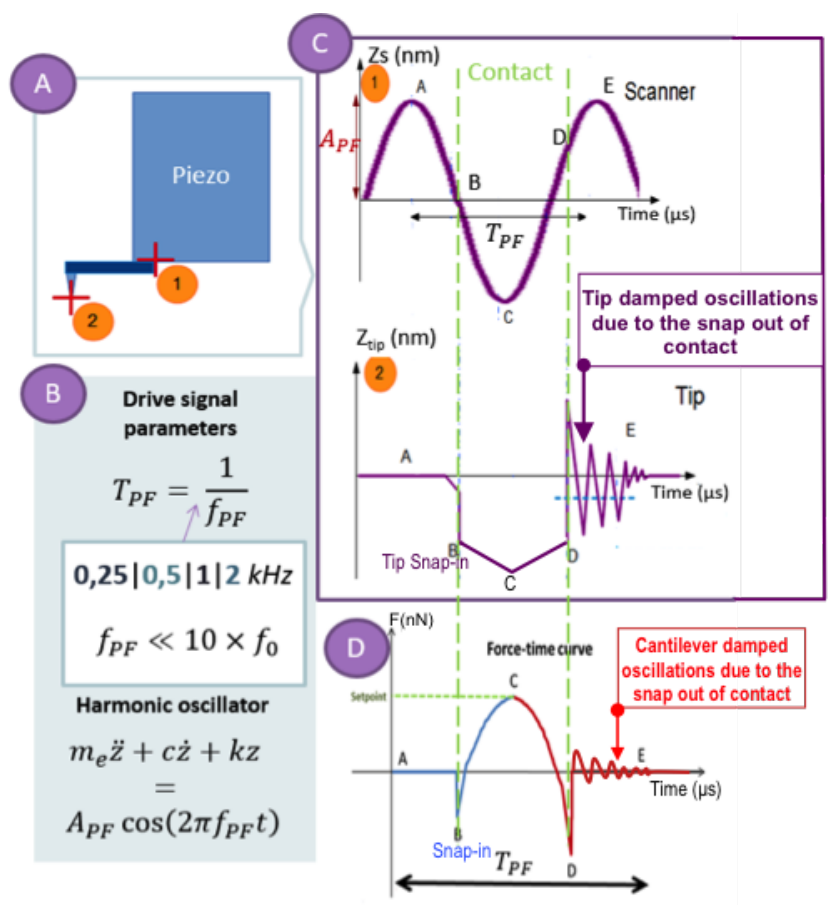

Fig. 4. (A) The piezoelectric scanner drives the clamp part of the cantilever (point A1) while the detection system records the deformation of the free end of the cantilever (point A2). (B) Signal excitation characteristics required to drive the cantilever out of its resonance $f_{0} ; m_{e}$ and care respectively the effective mass of the cantilever and the damping factor due to the fluid surrounding $(\mathrm{C} 1)$; A sinusoidal signal (frequency $f_{P F}$ and amplitude $A_{P F}$ ) is applied on the piezoelectric scanner to move up and down the cantilever, (C2) Tip position signal is recorded during the sinusoidal movement; (D) Full force-time curve recorded through the cantilever deformation : the approach curve (blue) exhibits the snap-in to contact (point $B$ ) following by the indentation phase reaching its maximum at the setpoint (phase $B C$ ) following by the retract curve (red) where the tip-sample force decreases until the snap-out of contact (point $D$ ).

liquid, we fixed the PF setpoint and frequency respectively to around ten of $\mathrm{nN}$ and $0.25 \mathrm{kHz}$. In fact, since the resonance frequency of the cantilever $f_{0}$ is shifted to a lower value in liquid than in air due to the drag effect [3], drive frequency has to decrease to avoid any cantilever oscillation. In spite of this precaution, some damped oscillations may appear when the tip suddenly snap out of the contact (Fig. 4C2) due to the kinetic energy and the non-zero quality factor of the cantilever. For the present study, we used the PF mode on an ICON AFM with Nanoscope Analysis software (version 8.15) in liquid (PBS solution). We also activated the PeakForce Capture option to get the raw force curves for each pixel of the image. Then, we applied a custom processing for the extraction of the contact stiffness $k_{C}$ from the slope of the unload curve by combining the force curve data, notably the peak force and the indentation depth (Fig. $3 \mathrm{~B})$. The results were exported towards the haptic interface in two separate matrices of $\left(256^{* 256)}\right.$ elements containing the $h$ indentation depth profile and the $k_{C}$ stiffness of a cell.

\section{HAPTIC IMPLEMENTATION}

The haptic implementation consists, in our case, to simulate at a macroscopic level the mechanical characteristics of the surface of a mouse osteoblast cell which was scanned using the AFM PF mode (Fig. 1B). To this aim, we had to develop a contact model that takes into account the main mechanical characteristics of the cell and then implement the application in a $3 \mathrm{D}$ engine.

\section{A. Mechanical Model}

The contact model is based on the assumption that the explored surface behaves as a viscoelastic material, which relevant to describe a spread cell [26]; [27]. According to the Kelvin-Voigt model, this kind of material can be represented by a purely elastic spring (stiffness) connected in parallel with a purely viscous damper (viscosity). Note that the surface is explored not only in depth ( $z$ axis), but also laterally ( $x, y$ axes), we thus have to develop a 3dimensional viscoelastic contact model. The 3-dimensional

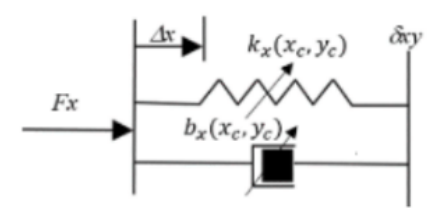

Fig. 5. The viscoelastic contact model used for the implementation of the haptic device. Note that the two other axis are similar.

contact model is shown in Fig. 5, where $F x, F y$, and $F z$ are the force components applied to the surface. In return, where is contact, the material reacts according to the mechanical characteristics (elasticity and viscosity). This reaction is traduced by the haptic device thanks to a 3-dimensional returning force according to the sample local stiffness values and a chosen damping coefficient. Consequently, the operator gets a sensory feeling of the explored material. In our notations, $\Delta x, \Delta y$ and $\Delta z$, represents the displacement into the material, we have :

$$
\Delta x=x-x_{c} \quad ; \quad \Delta y=y-y_{c} \quad ; \quad \Delta z=z-h_{x y}
$$

where $(x, y, z)$ represents the position of the moving part of the haptic device, $\left(x_{c}, y_{c}\right)$ are the coordinates of the contact point, and $h_{x y}$ is the reconstructed surface profile at the contact point. In our application, the mechanical characteristics are not homogeneous in space, i.e. the stiffness coefficients $k_{x}, k_{y}, k_{z}$, and the viscous coefficients $b_{x}, b_{y}, b_{z}$, depend on the contact position $\left(x_{c}, y_{c}\right)$. However, for small displacements, it can be assumed that these coefficients are equals and constants in the vicinity of the contact position, giving thus :

$$
\begin{gathered}
k_{x}\left(x_{c}, y_{c}\right)=k_{y}\left(x_{c}, y_{c}\right)=k_{z}\left(x_{c}, y_{c}\right)=k_{c}\left(x_{c}, y_{c}\right) \\
b_{x}\left(x_{c}, y_{c}\right)=b_{y}\left(x_{c}, y_{c}\right)=b_{z}\left(x_{c}, y_{c}\right)=b\left(x_{c}, y_{c}\right)
\end{gathered}
$$

Under this assumption, the algorithm will only require the sample stiffness and viscous coefficients matrices $k_{C}$ and $b$ as well as the sample profile $h_{x y}$ at the contact point $\left(x_{c}\right.$, $\left.y_{c}\right)$. These aspects have been discussed in Section 3 except for the $b$ values which will be given a constant default value to ensure the haptic feedback stability. 


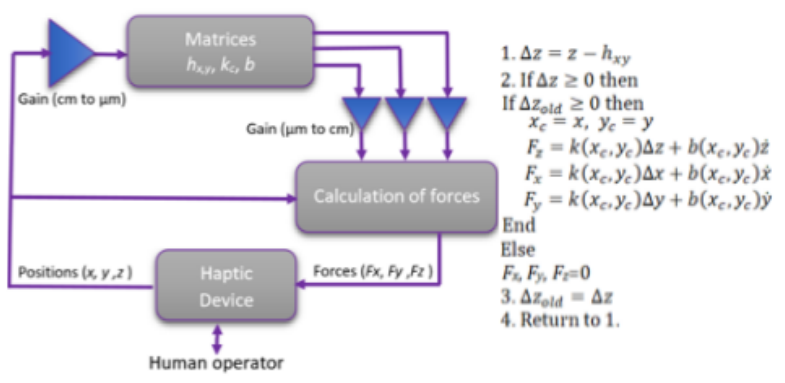

Fig. 6. Schematic diagram of the force feedback loop implemented to produce the haptic rendering.

\section{B. Novint device}

The Novint Falcon haptic device is a 3D haptic controller that produces a return effort according to the operator arm displacement. The returned forces can be calculated according to the considered application. In our case, these forces are calculated to reproduce the mechanical properties of the explored cell recorded by AFM. Fig. 6 shows the general principle used to this end. The calculation of the haptic forces has been performed according to the algorithm described in Fig. 6 and running at $1 \mathrm{kHz}$.

\section{Software implementation and Results}

The AFM topography and stiffness maps were initially exported from the Nanoscope Analysis software towards MATLAB where were Gaussian-filtered and plotted. The two $(256 * 256)$ elements matrices of two different samples are displayed in Fig. 7 : on the top, a mixture of two polymers where Low Density Polyethylene (LPDE) microspheres are embedded in a matrix of polystyrene (PS), on the bottom the fixed osteoblast cell on Fn micropattern with a $I$ shape. The PS/LDPE is a reference sample with well-known mechanical properties (ratio of 10 between the two Young Modulus). We used it as a test sample to validate the PF mode implementation in the interactive virtual nanoscene coupled with the Novint haptic device (Fig. 8). Notice that the scanned image of the cell (Fig. $7 \mathrm{c}$ ) is $\left(45^{*} 45\right) \mu \mathrm{m}$, the nucleus maximum height is $2.0 \mu \mathrm{m}$ and the thickness of the lateral focal adhesions is between 0.02 and $0.2 \mu \mathrm{m}$. As for the stiffness, we may notice values in the central region between 0.06 and $0.12 \mathrm{~N} / \mathrm{m}$, showing that the nucleus is the softest region. The focal adhesions and the stress fibers of the cytoskeleton, are between 0.12 to $0.25 \mathrm{~N} / \mathrm{m}$ and the substrate is around 0.4 $\mathrm{N} / \mathrm{m}$. We may also notice that the focal adhesions in the right corner are almost invisible on the topography map in the Fig. 7c (hidden by the nucleus), while they are quite noticeable in the stiffness map (Fig. 7d). This underlines the interest of combination of sensorial renderings to display two different sample information : thanks to the haptic exploration we could "sense" the cell mechanics beyond the visible perception of its morphology. Next step was to transform the $h_{x y}$ matrix in a $3 \mathrm{D}$ object such as a «.stl » file type, which was straightforward to convert in MATLAB by using a function called «surf 2 stl ». The $k_{C}$ matrix is
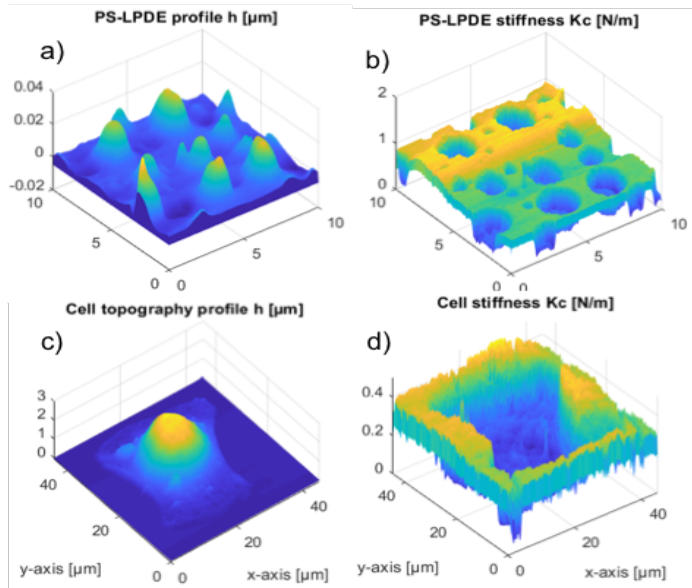

Fig. 7. (a) and (c) Surface plots of the topography matrix $h_{x y}$ and associated stiffness matrix $k_{C}$ (c) and (d) of respectively PS/LDPE sample and osteoblast cell fixed on a $I$ shape micro-pattern obtained in fluid (air for PS/LDPE, water for the cell) by AFM PF mode with a setpoint of 15 $\mathrm{nN}, f_{P F}=1 \mathrm{kHz}, A_{P F}=300 \mathrm{~nm}$, scan rate of $0.5 \mathrm{~Hz}$.

saved in a «.csv » file. Once the «.stl » file of the scanned cell is generated, it will be converted into an «.obj» file using an online 3D files converter such as meshconvert.com. The converted object file is then imported into the Unity engine whose interface looks like in Fig. 8. The converted model is initially unscaled. For the cell a modification of scale and orientation is performed using a fixed gain of $0.22 \mathrm{~cm} / \mu \mathrm{m}$ to adapt the cell $3 \mathrm{D}$ object which is $45 \mu \mathrm{m}$ to the size of the $10 \mathrm{~cm}$ workspace of the haptic device.For the PS/LPDE sample the $X Y$ gains are $1 \mathrm{~cm} / \mu \mathrm{m}$ while the vertical profile was increased to $15 \mathrm{~cm} / \mu \mathrm{m}$ for a better view. A set of $\mathrm{C} \sharp$ scripts were developed in Unity to integrate the Novint haptic interaction in Virtual Reality. The first one is the communication script that gets the $(x, y, z)$ positions of the haptic device and restores it to the tip on the $3 \mathrm{D}$ virtual environment. The second script allows the physical interaction between the $3 \mathrm{D}$ objects using mesh colliders. The principle is to detect collision between the tip and the surface $\left(x_{c}, y_{c}\right)$ and generate forces $(F x, F y, F z)$ on the haptic device to react to the movement by up to $4 \mathrm{~N}$ of net force. For this purpose, a separate script extracts the local stiffness value from the stiffness matrix $k_{C}$ which was saved into a «.csv » file by using a parser. The AFM tip is controlled by the haptic device and it appears as a cone in the virtual environment (Fig. 8a). When this tip apex enters on collision with the surface its color turns red upon indenting the cell. This color change is useful to highlight the experimenter that the indentation process starts. For a better 3D immersion in the VR environment we used two slightly-shifted virtual cameras in a stereoscopic configuration and connected to an Oculus Rift headset (Fig. 8c), whose configuration is straightforward using the provided Unity plug-in.

\section{CONCLUSION}

The interest of our proposal concerns the specific contribution of the nano-rheological approach to functionalize 


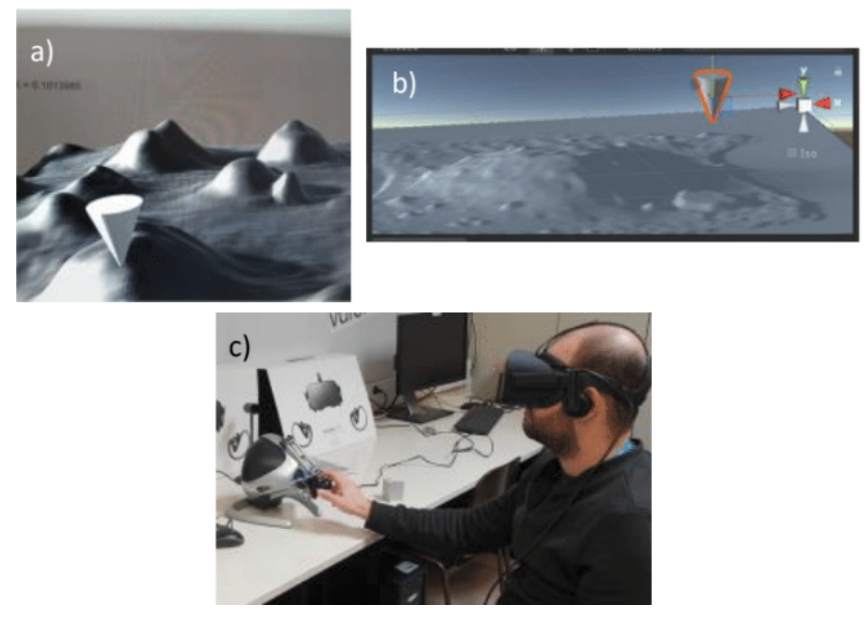

Fig. 8. The immersive application using Oculus Rift VR headset applied to LDPE/PS sample (a) and to the fixed cell (b); (c) Through the Novint Falcon haptic device, the user controls tip (cone shape) and feels the local sample elasticity

the image of the cell and its related architectural components by mechanical data. Then, to perceive this image by a simple haptic device accessible to students coming from very different knowledge background. The tactilomechanical perception of a micrometric cell derived from the living is often a revelation for the students. Biologists realize that the structure of the cellular skeleton composed of polymers in self-equilibrium, is closely related to the cellular mechanical properties of internal tension, stiffness, and adhesion. Physicists discover how the forms of life are « diagrams of the underlying forces » and that the living at the scale of the cell and the molecule can be described and experience by the action of a force on a mass.

\section{ACKNOWLEDGMENT}

National project FUI -REVE 5D : Réalité Augmentée pour la Culture, les Infrastructures et l'Industrie. CIME Nanotech for AFM support and funding of Claudie Petit.

\section{REFERENCES}

[1] K. Jansen, P. Atherton, and C. Ballestrem, "Mechanotransduction at the cell-matrix interface," Seminars in Cell \& Developmental Biology, vol. 71, pp. 75 - 83, 2017.

[2] N. Eroshenko, R. Ramachandran, V. K. Yadavalli, and R. R. Rao, "Effect of substrate stiffness on early human embryonic stem cell differentiation," Journal of Biological Engineering, vol. 7, no. 1, p. 7, Mar 2013.

[3] M. Rauzi, P. Verant, T. Lecuit, and P.-F. Lenne, "Nature and anisotropy of cortical forces orienting drosophila tissue morphogenesis," Nature Cell Biology, vol. 10, pp. 1401-, Nov. 2008.

[4] M. Lekka, "Discrimination between normal and cancerous cells using afm," BioNanoScience, vol. 6, no. 1, pp. 65-80, Mar 2016.

[5] N. Wang, "Review of cellular mechanotransduction," Journal of Physics D : Applied Physics, vol. 50, no. 23, p. 233002, may 2017.

[6] P. Bivall, S. Ainsworth, and L. A. E. Tibell, "Do haptic representations help complex molecular learning ?" Science Education, vol. 95, no. 4, pp. 700-719, 2011.

[7] G. Millet, A. Lécuyer, J.-M. Burkhardt, S. Haliyo, and S. Régnier, "Haptics and graphic analogies for the understanding of atomic force microscopy," International Journal of Human-Computer Studies, vol. 71 , no. 5 , pp. $608-626,2013$.
[8] C. Basdogan, M. Sedef, M. Harders, and S. Wesarg, "Vr-based simulators for training in minimally invasive surgery," IEEE Computer Graphics and Applications, vol. 27, no. 2, pp. 54-66, March 2007.

[9] J. Minogue, M. Gail Jones, B. Broadwell, and T. Oppewall, "The impact of haptic augmentation on middle school students' conceptions of the animal cell," Virtual Reality, vol. 10, no. 3, pp. 293-305, Dec 2006.

[10] H. Ladjal, J. Hanus, A. Pillarisetti, C. Keefer, A. Ferreira, and J. P. Desai, "Realistic visual and haptic feedback simulator for real-time cell indentation," in 2010 IEEE/RSJ International Conference on Intelligent Robots and Systems, Oct 2010, pp. 3993-3998.

[11] S. Marliere, F. Marchi, J. L. Florens, A. Luciani, and J. Chevrier, "An augmented reality nanomanipulator for learning nanophysics : The "nanolearner" platform," in 2008 International Conference on Cyberworlds, Sep. 2008, pp. 94-101.

[12] H.-J. Butt, B. Cappella, and M. Kappl, "Force measurements with the atomic force microscope : Technique, interpretation and applications," Surface Science Reports, vol. 59, no. 1, pp. 1 - 152, 2005.

[13] K. Radotić, C. Roduit, J. Simonović, P. Hornitschek, C. Fankhauser, D. Mutavdžić, G. Steinbach, G. Dietler, and S. Kasas, "Atomic force microscopy stiffness tomography on living arabidopsis thaliana cells reveals the mechanical properties of surface and deep cell-wall layers during growth," Biophysical Journal, vol. 103, no. 3, pp. 386 - 394, 2012.

[14] L. Lu, S. J. Oswald, H. Ngu, and F. C.-P. Yin, "Mechanical properties of actin stress fibers in living cells," Biophysical journal, vol. 95, no. 12, pp. 6060-6071, 2008.

[15] B. Pittenger, N. Erina, and C. Su, Mechanical Property Mapping at the Nanoscale Using PeakForce QNM Scanning Probe Technique. Dordrecht : Springer Netherlands, 2014, pp. 31-51.

[16] A. Calzado-Martín, M. Encinar, J. Tamayo, M. Calleja, and A. San Paulo, "Effect of actin organization on the stiffness of living breast cancer cells revealed by peak-force modulation atomic force microscopy," ACS Nano, vol. 10, no. 3, pp. 3365-3374, 2016, pMID : 26901115.

[17] H. Schillers, I. Medalsy, S. Hu, A. L. Slade, and J. E. Shaw, "Peakforce tapping resolves individual microvilli on living cells," Journal of Molecular Recognition, vol. 29, no. 2, pp. 95-101, 2016.

[18] G. Binnig, C. F. Quate, and C. Gerber, "Atomic force microscope," Phys. Rev. Lett., vol. 56, pp. 930-933, Mar 1986.

[19] E. Meyer, H. J. Hug, and R. Bennewitz, Introduction to Scanning Probe Microscopy. Berlin, Heidelberg : Springer Berlin Heidelberg, 2004.

[20] R. Lévy and M. Maaloum, "Measuring the spring constant of atomic force microscope cantilevers : thermal fluctuations and other methods," Nanotechnology, vol. 13, no. 1, pp. 33-37, dec 2001.

[21] P. K. Hansma, J. P. Cleveland, M. Radmacher, D. A. Walters, P. E. Hillner, M. Bezanilla, M. Fritz, D. Vie, H. G. Hansma, C. B. Prater, J. Massie, L. Fukunaga, J. Gurley, and V. Elings, "Tapping mode atomic force microscopy in liquids," Applied Physics Letters, vol. 64,

[22] G. AU Thomas, N. A. AU Burnham, T. A. AU Camesano, and Q. AU Wen, "Measuring the mechanical properties of living cells using atomic force microscopy," JoVE, no. 76, pp. e50 497-, 2013.

[23] L. Sirghi, "Atomic force microscopy indentation of living cells," $\mathrm{Mi}$ croscopy : science, technology. Applications and Education, Formatex, Badajoz, pp. 433-440, 2010.

[24] D. J. Shuman, A. L. Costa, and M. S. Andrade, "Calculating the elastic modulus from nanoindentation and microindentation reload curves," Materials Characterization, vol. 58, no. 4, pp. 380 - 389, 2007.

[25] B. Foster, "New atomic force microscopy(afm) approaches life sciences gently, quantitatively, and correctively," American laboratory, vol. 44, no. 4, pp. 24-28, 2012.

[26] S. Féréol, R. Fodil, V. Laurent, E. Planus, B. Louis, G. Pelle, and D. Isabey, "Mechanical and structural assessment of cortical and deep cytoskeleton reveals substrate-dependent alveolar macrophage remodeling," Bio-medical materials and engineering, vol. 18, no. s1, pp. 105-118, 2008.

[27] R. Fodil, V. Laurent, E. Planus, and D. Isabey, "Characterization of cytoskeleton mechanical properties and 3d-actin structure in twisted adherent epithelial cells," Biorheology, vol. 40, 2003. 